\title{
Les mécanismes de la transduction du signal par le récepteur de la prolactine
}

La prolactine (PRL), hormone d'origine hypophysaire, apparaît comme un puissant immunorégulateur. Son récepteur (PRLR) appartient à la nouvelle superfamille, récemment décrite, des récepteurs des cytokines, de l'hormone de croissance $(\mathrm{GH})$ et de PRL [1]. Comme tous les membres de cette famille, le domaine extracellulaire du PRLR possède une région d'environ 200 acides aminés que l'on peut diviser en deux parties de 100 acides aminés chacune, une région $\mathrm{N}$-terminale de structure similaire à celle des immunoglobulines, contenant quatres résidus cystéine très conservés entre les différents membres, et une région Cterminale, de structure similaire à celle de la fibronectine de type III, contenant une cinquième cystéine ainsi que le motif WSXWS*, conservé chez tous les membres de la famille, excepté le récepteur de $\mathrm{GH}$, GHR [2].

Ces récepteurs ne présentent pas ou peu de similarités dans leur domaine cytoplasmique, bien que certaines régions soient restées conservées, particulièrement dans la région proximale de la membrane, et cela dans l'ensemble des espèces étudiées à ce jour. De plus, à la différence des récepteurs des facteurs de croissance, les membres de la famille des récepteurs de cytokines/GH/PRL ne possèdent pas d'activité tyrosine kinase intrinsèque, ni de séquence consensus pour la fixation de l'ATP/GTP. En revanche, ils sont capables de s'associer à des protéines tyrosine kinases intracellu-

\section{TIRÉS A PART}

laires de la famille des Jak et des Src kinases $\left(m / s n^{\circ} 2\right.$, vol. 10, p. 202, [1]). Les Jak kinases ne possèdent pas de domaine de fixation du ligand $\mathrm{ni}$ de domaine transmembranaire, et présentent deux domaines de type kinase. On a montré qu'elles étaient associées à plusieurs des membres de la famille des récepteurs des cytokines/GH/PRL et à l'origine des toutes premières étapes d'activation de la transmission du signal.

Les données établies concernant la transmission du signal du PRLR sont présentées dans la figure 1. Nous avons montré que le PRLR est associé à la tyrosine kinase Jak2 et que l'activation de l'enzyme par les hormones lactogènes induit la phosphorylation de ses résidus tyrosine [3-5]. Cette association entre le PRLR et Jak2, est constitutive, comme cela a également été montré pour plusieurs autres récepteurs de cette même famille. L'activation de la phosphorylation, très rapide, représente un des tout premiers événements impliqués dans la transmission du signal du PRLR.

L'oligomérisation représente un processus clé pour la transduction du signal par les récepteurs de cette famille qui, selon les cas, s'associent en hétérodimères ou en homodimères [1]. Jusqu'à présent, seul le modèle du récepteur $\mathrm{GH}$ a été bien étudié ; la molécule $G H$ possède deux sites de fixation au récepteur, et fixe une première molécule de récepteur sur son site 1, puis accroche ensuite une seconde molécule de récepteur sur son site 2, induisant ainsi la formation d'un homodimère de GHR [6]. Cette activation est séquentielle, puisque des mutants du site 1 de la $\mathrm{GH}$ ne sont pas capables de se lier au récepteur ni de transmettre le signal, alors que des mutants du site 2 de la $\mathrm{GH}$ se lient au récepteur mais sont des antagonistes qui s'opposent aux effets de l'hormone [7]. Cela semble aussi être le cas du récepteur PRL, puisque la $\mathrm{GH}$ humaine, qui se lie (en présence de zinc) au PRLR, induit la prolifération des cellules Nb2**, avec des profils d'activité des mutants des sites 1 et 2 identiques à ceux précédemment décrits [8]. D'autre part, des anticorps monoclonaux dirigés contre le domaine extracellulaire du PRLR, mais pas leurs fragments Fab monovalents, sont capables de mimer les effets de la prolactine sur la prolifération des cellules Nb2 ainsi que sur l'induction des gènes des protéines du lait (référencés dans [9]).

Nous avons pu montrer, grâce à l'utilisation des anticorps monoclonaux et des fragments Fab monovalents, que la bivalence du ligand était nécessaire pour induire, non seulement la prolifération cellulaire, mais aussi l'activation de la phosphorylation de Jak2 [9]. Ces résultats suggèrent par là même que la dimérisation du PRLR, en réponse à la liaison du ligand, représente la première étape de l'action hormonale dans l'activation des événements précoces, aussi bien que tardifs, observés dans la signalisation cellulaire du PRLR (figure 1). Cette dimérisation du récepteur induit un changement de conformation du PRLR et des

\footnotetext{
* Trp-Ser-n 'importe quel acide aminé-Trp-Ser. ** Les cellules Nb2 sont des cellules $T$ de rat prélymphomateuses.
} 
molécules associées, activant ainsi le site catalytique de la tyrosine kinase Jak2.

Grâce à une technique développée au laboratoire, consistant à marquer spécifiquement les récepteurs exprimés à la surface cellulaire au moyen d'un anticorps monoclonal biotinylé, nous avons démontré que l'activation de Jak2 entraîne la phosphorylation des résidus tyrosine présents sur la kinase et sur le PRLR luimême [4]. Ces phosphorylations représentent une étape importante dans la transduction du signal. En effet, les tyrosines phosphorylées, et les courtes séquences qui les entou- rent, sont des zones de reconnaissance pour les domaines SH2 des molécules transductrices, pouvant servir de point d'ancrage à ces molécules signalisatrices et initier ainsi l'activation de la transduction [10] (figure 1). Il faut à cet égard souligner que les voies de transduction reliées aux phosphotyrosines de cette kinase sont probablement communes à l'ensemble des récepteurs de la superfamille auxquels elle se trouve associée, alors que celles engendrées par les résidus phosphorylés présents sur le récepteur exprimeront une certaine spécificité d'action.

Le fait que Jak2 soit constitutive-

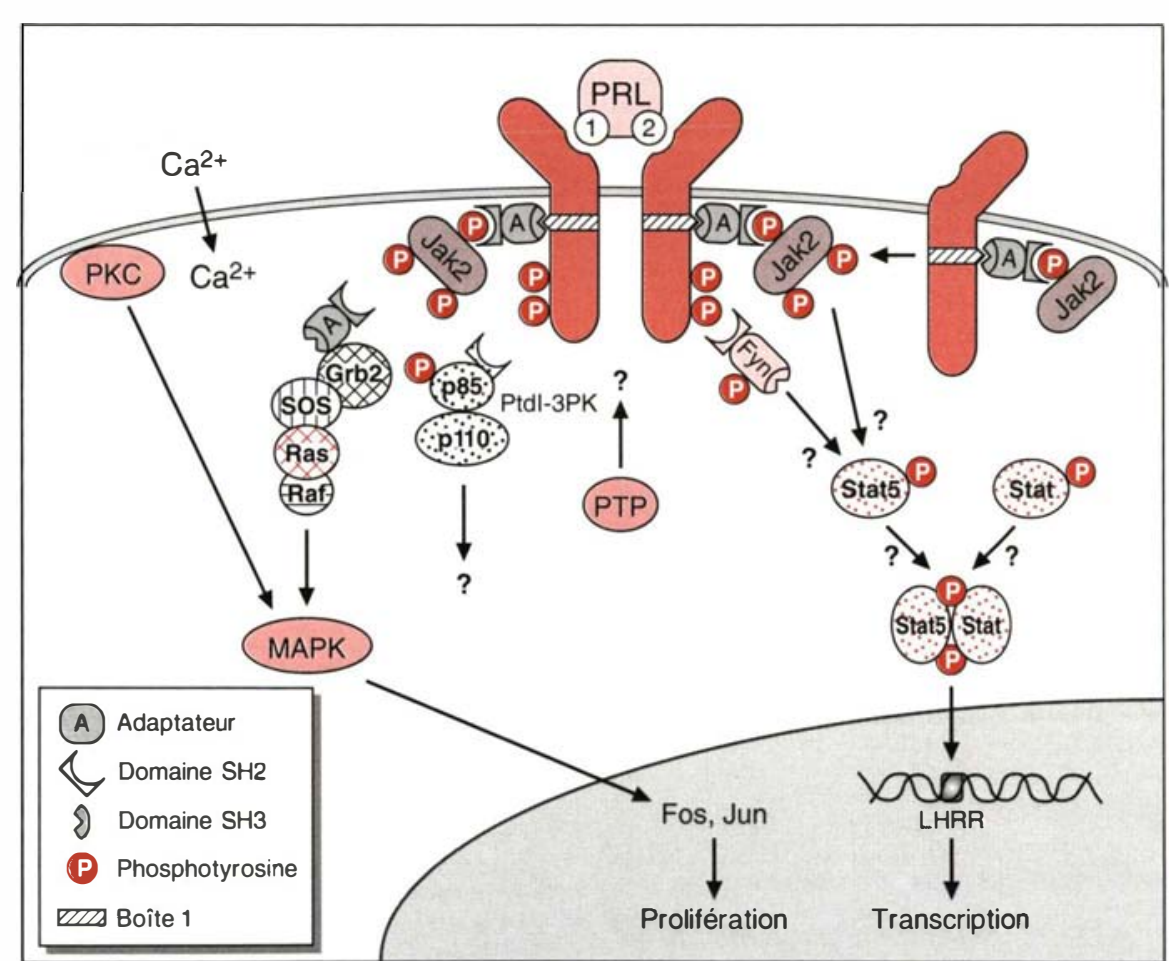

Figure 1. Représentation schématique des différentes voies de transduction du signal du récepteur de la prolactine. La prolactine avec ses deux sites de fixation du récepteur induit la dimérisation du PRLR, engendrant ainsi les différentes voies $d^{\prime}$ activation de la transduction. Parmi celles-ci, on retrouve la voie d'activation des MAP kinases (MAPK), via une molécule adaptatrice (A) contenant des domaines $\mathrm{SH} 2$ et SH3, Grb2, SOS, Ras, Raf. La phosphatidylinositol 3-phosphate kinase (Ptdl-3PK) est elle aussi activée en réponse à la stimulation par la prolactine, mais son rôle est encore inconnu. L'activation des tyrosine kinases Jak2 et Fyn permet la phosphorylation des autres molécules transductrices à l'intérieur de la cellule. Enfin, il reste à déterminer la ou les protéines phosphatases impliquées dans la déphosphorylation de toutes ces molécules. LHRR : lactogen hormone response region; PTP: phosphotyrosine phosphatase. ment associé à plusieurs récepteurs de cette famille, incluant le PRLR, suggère l'existence d'une région d'association avec la kinase, commune à l'ensemble de ces récepteurs [11]. Seule la région cytoplasmique proche de la membrane présente des similarités entre les différents récepteurs, cette région comportant en particulier une courte séquence de 8 acides aminés (boîte 1 ) riche en proline, qui apparaît indispensable à la transduction du signal. A l'aide de mutants de délétion touchant le domaine cytoplasmique du PRLR, nous avons mis en évidence la nécessité de cette boîte 1 pour l'association avec Jak2. Cependant, cette association n'est pas suffisante pour induire l'activation de la transcription des gènes des protéines du lait, suggérant par là même un rôle des résidus tyrosine, présents dans la partie carboxy-terminale du récepteur, dans la transduction du signal [12].

Si l'association des Jak kinases avec les récepteurs de la superfamille des récepteurs de cytokines semble maintenant évidente, d'autres tyrosine kinases sont aussi impliquées. De nombreux travaux indiquent que des membres de la famille des Src kinases jouent un rôle dans ces interactions [1]. Récemment, il a été montré que la tyrosine kinase Fyn est également associée au PRLR [13]. Il reste toutefois à déterminer quel est son site d'interaction avec le récepteur, ainsi que ses substrats. Il est possible que Fyn se fixe sur les résidus tyrosine présents dans la partie C-terminale du PRLR, suggérant ainsi un rôle de cette kinase dans l'activation de facteurs transcriptionnels spécifiques. A ce niveau, peu de choses sont connues, cependant la région proximale du promoteur du gène codant pour la $\beta$-caséine, inductible par la prolactine, contient une séquence de réponse aux hormones lactogènes (lactogen hormone response region, LHRR). Le facteur protéique responsable de l'activation de la transcription du gène de la $\beta$ caséine, associé sous forme d'homoou d'hétérodimère à ces séquences LHRR (figure 1), est le facteur de croissance de la glande mammaire ou MGF (mammary gland factor). 
L'ADNc de ce facteur a récemment été cloné [14] et présente des homologies de séquence avec les membres de la famille des protéines Stat qui sont des facteurs transcriptionnels impliqués dans la signalisation des récepteurs hématopoïétiques mais aussi des récepteurs tyrosine kinases $[1,11]$. Le MGF est actuellement considéré comme un nouveau membre de cette famille (Stat5) et pourrait être activé, directement ou indirectement, par la tyrosine kinase Fyn (figure 1).

Cela ne représente qu'une partie des différentes voies de transduction du PRLR. En effet, des résultats préliminaires indiquent que la phosphatidylinositol 3-kinase, la voie d'activation des MAP kinases, ainsi que certaines protéines phosphatases (figure 1) pourraient, elles aussi, être impliquées dans la transduction du signal du PRLR (résultats non publiés). Enfin, la prolactine augmente la concentration intracellulaire en $\mathrm{Ca}^{2+}$, par un mécanisme n'impliquant pas l'inositol triphosphate [15], et peut aussi induire la prolifération cellulaire via l'activation de la protéine kinase $\mathrm{C}$.

En conclusion, les différentes voies de transduction du signal du récepteur de la prolactine, résumées dans la figure 1, apparaissent très similaires à celles observées pour les autres membres de la superfamille des récepteurs des cytokines/GH/PRL, impliquant à la fois des processus de dimérisation des récepteurs, et l'association avec des tyrosine kinases cytoplasmiques (Jak, Src) ainsi que des facteurs transcriptionnels (STAT) communs

\section{TIRÉS A PART}

\section{RÉFÉRENCES}

1. Dusanter I, Mayeux P, Gisselbrecht S. Transduction du signal par les récepteurs de cytokines, médecine/sciences $1994 ; 10$ : 825-35.

2. Bazan JF. Structural design and molecular evolution of a cytokine receptor superfamily. Proc Natl Acad Sci USA 1990; 87 : 6934-8.

3. Rui H, Kirken RA, Farrar WL Activation of receptor-associated tyrosine kinase Jak2 by prolactin. $J$ Biol Chem $1994 ; 269: 53648$.

4. Lebrun IJ, Ali S, Sofer L, Ullrich A, Kelly $\mathrm{PA}$. Prolactin induced proliferation of $\mathrm{Nb2}$ cells involves tyrosine phosphorylation of the prolactin receptor and its associated tyrosine kinase Jak2. J Biol Chem $1994 ; 269$ : 14021-6.

5. Dusanter-Fourt I, Muller O, Ziemiecki A, Mayeux P, Drucker B, Djiane J, Wilks A, Harpur AG, Fisher S, Gisselbrecht S. Identification of Jak protein tyrosine kinases as signaling molecules for prolactin. Functional analysis of prolactin receptor and prolactin - erythropoietin receptor chimera expressed in lymphoid cells. EMBO J 1994 . $13: 2583-91$

6. Cunningham BC, Ultsch $\mathrm{M}$, de Vos AM, Mulkerrin MG, Wells JA. Dimerization of the extracellular domain of the human growth hormone receptor by a single hormone molecule. Science 1991 ; 254 : 821-5.

7. Fuh G, Cunningham BC, Fukunaga R Goeddel DV, Wells JA, Rational design of potent antagonists to the human growth hormone receptor. Science $1992 ; 256$ : $1677-80$

8. Fuh G, Colosi P, Wood WI, Wells JA Mechanism-based design of prolactin receptor antagonists. I Biol Chem 1993: $268: 5376-81$.

9. Rui H, Lebrun J, Kirken RA, Farrar WL Jak2 activation and cell proliferation induced by antibody-mediated prolactin receptor dimerization. Endocrinology 1994 (sous presse).

10. Chardin P. Domaines $\mathrm{SH}_{2}$ et $\mathrm{SH} 3$. médecine/sciences $1994 ; 10$ : 709-12.

11. Kahn A. De la membrane au noyau, un couplage direct entre des récepteurs de cytokines et la machinerie transcriptionnelle. médecine/sciences $1994 ; 10$ : 202-5.

12. Lebrun IJ, Ali S, Ullrich A, Kelly PA. Proline-rich sequence-mediated Jak2 association to the prolactin receptor is required but not sufficient for signal transduc tion. 1994 (sous presse)

13. Clevenger CV, Medaglia MV. The protein tyrosine kinase p59fyn is associated with prolactin (PRL) receptor and is activated by PRL stimulation of T-lymphocytes. Mol Endocrinol $1994 ; 8: 674-81$.

14. Wakao H, Gouilleux F, Groner B. Mammary gland growth factor (MGF) is a novel member of the cytokine regulated transcription factor gene family and confers the prolactin response. $E M B O J 1994 ; 13$ : $2182-91$.

15. Vacher P, Tran Van Chuoi M, Paly J Djiane J, Dufy B. Short term effect of prolactin on intracellular calcium in chinese hamster ovary cells stably transfected with prolactin receptor complementary desoxyribonucleic acids. Endocrinology $1994 ; 134$ : 1213-8.

Jean-Jacques Lebrun

Paul A. Kelly

Inserm U. 344, endocrinologie moléculaire, faculté de médecine Necker-EnfantsMalades, 156, rue de Vaugirard, 75730 Paris Cedex 15, France. 